\title{
Electronic and surface properties of PbS nanoparticles exhibiting efficient multiple exciton generation
}

\author{
Samantha J. O. Hardman, ${ }^{* a}$ Darren M. Graham, ${ }^{a}$ Stuart K. Stubbs, ${ }^{a}$ Ben F. Spencer, ${ }^{a, b}$ Elaine A. \\ Seddon, ${ }^{a}$ Ho-Ting Fung, ${ }^{a}$ Sandra Gardonio, ${ }^{c \neq}{ }^{p}$ Fausto Sirotti, ${ }^{d}$ Mathieu G. Silly, ${ }^{d}$ Javeed Akhtar, ${ }^{e \S}$ Paul \\ ${ }_{5}$ O'Brien, ${ }^{e}$ David J. Binks ${ }^{a}$ and Wendy R. Flavell. ${ }^{a}$
}

\author{
Received Xth $X X X X X X X X X 2011$, Accepted $X$ th $X X X X X X X X X 20 X X$ \\ DOI: $10.1039 / b 000000 x$
}

Ultrafast transient absorption measurements have been used to study multiple exciton generation in solutions of $\mathrm{PbS}$ nanoparticles vigorously stirred to avoid the effects of photocharging. The threshold and 10 slope efficiency of multiple exciton generation are found to be $2.5 \pm 0.2 \times \mathrm{E}_{\mathrm{g}}$ and $0.34 \pm 0.08$, respectively. Photoemission measurements as a function of nanoparticle size and ageing show that the position of the valence band maximum is pinned by surface effects, and that a thick layer of surface oxide is rapidly formed at the nanoparticle surfaces on exposure to air.

\section{Introduction}

15 The theoretical maximum efficiency of single junction solar cells under unconcentrated sunlight is currently limited to ca. $30 \%$ by the rapid cooling of the hot carriers produced by the absorption of photons with energy in excess of the band gap. ${ }^{1}$ However, photovoltaic (PV) devices based on nanoparticles (NPs) may be

20 able to exceed this limit by making use of multiple exciton generation (MEG), also known as carrier multiplication (CM), where the excess energy of an absorbed photon is used to generate extra electron-hole pairs rather than being wasted as heat. MEG has been observed spectroscopically in a number of 25 different NPs including $\mathrm{CdSe},{ }^{2} \mathrm{CdTe},{ }^{3} \mathrm{CdSe} / \mathrm{CdTe},{ }^{4} \mathrm{InP},{ }^{5} \mathrm{InAs},{ }^{6}$ $\mathrm{PbSe}^{7}{ }^{\mathrm{PbS}},{ }^{7} \mathrm{PbTe},{ }^{8}$ and $\mathrm{Si}^{9}{ }^{9}$ Moreover, photoconductive ${ }^{10}$ and photovoltaic $^{11}$ devices incorporating PbS NPs which exhibit MEG have also been reported recently, and PbS NPs are the subject of the study presented here. Previous investigations of 30 MEG in PbS NPs ${ }^{7,12-14}$ have reported quantum yield (QY) values which differed significantly, varying from $\sim 110 \%$ to $\sim 280 \%$ for approximately the same ratio of photon energy $(\mathrm{h} v)$ to band gap $\left(E_{g}\right)$. Most of these studies were conducted before it became apparent that photocharging of samples can lead to erroneous QY 35 values because the spectroscopic signature of a single exciton in a charged NP is similar to that of biexciton in a neutral NP. ${ }^{15-17}$ However, it has recently been shown that rapidly stirring ${ }^{15,16}$ or flowing ${ }^{17}$ a solution of NPs can prevent this effect, leading to reliable QY values. A very recent study ${ }^{14}$ was largely performed

40 on static samples of PbS NPs but did present a typical transient obtained when the sample was static and compared it to one obtained when the sample was flowing. From this comparison, the authors estimated that photocharging could account for $\sim 10 \%$ of the apparent QY in their samples.

45 The advantages of generating more than one electron-hole pair per incident photon through MEG are lost if those carriers cannot be extracted from the NP to contribute to a photocurrent. It is therefore important to determine the electronic structure and chemistry of the interface between the NP and its surroundings. ${ }_{50}$ In use in a device, the NPs are typically sandwiched between suitable photoanode and photocathode materials in a solid state device. As the size of a NP decreases its band gap increases, due to the quantum confinement effect, and the surface:volume ratio increases. Because small nanoparticles have such a high 55 surface:volume ratio the properties of this interface and of the NP surface may dominate the characteristics of the NP. X-ray photoelectron spectroscopy (XPS) is therefore a very suitable technique for the study of NPs, as it is sensitive to only the first few layers of a surface. There has been some investigation of the ${ }_{60}$ effects of surface chemistry on the properties of nanoparticle samples. ${ }^{18}$ It is now clear that the choice of capping ligand may affect not only the stability and solubility of the nanoparticles, but also the local vacuum level, ${ }^{19}$ and the optical properties of the sample, ${ }^{18}$ while the substrate upon which the nanoparticles are ${ }_{65}$ deposited may pin the energy of the highest occupied molecular orbital (HOMO) ${ }^{20}$ It is therefore clear that issues of surface chemistry and preparation have an important effect on the energy level line-up of the nanoparticle/substrate interface, a crucial parameter in PV design as it affects the efficiency of charge 70 extraction. ${ }^{21}$ Previous XPS studies of PbS nanoparticles have shown that the species present at the surface depends on the choice of ligand, ${ }^{22}$ and that oxidation products may be found at the surface. ${ }^{23}$ Indeed, in the case of PbSe nanoparticles this oxidation is found to transform $50 \%$ of the nanoparticle volume 75 within 24 hours. ${ }^{24}$ Surface states are predicted to have a strong effect on the rate of relaxation of hot carriers by impact ionisation, thus affecting $\mathrm{MEG},{ }^{25}$ although recent experiments on PbSe suggest that the effect on MEG efficiency is not marked. ${ }^{24}$ It seems clear, therefore, that it is important to probe not only 80 whether or not MEG can be observed, but also whether or not the 
surface and interface properties of the NPs allow MEG to be used. In this study we use ultrafast transient absorption spectroscopy to determine the threshold and slope efficiency of MEG in stirred solutions of PbS NPs. We then use photoemission 5 to investigate the electronic structure and surface composition of a range of $\mathrm{PbS}$ NPs that show efficient MEG, deposited on photoanode substrates. We investigate how the surface composition changes with exposure to air, and consider the implications for carrier dynamics.

10

\section{Experimental}

$\mathrm{PbS}$ NP samples were prepared by a novel environmentallybenign method, using olive oil as both solvent and capping agent, as described in detail elsewhere. ${ }^{23}$ Briefly, $\mathrm{PbO}$ and TMS 15 (bistrimethylsilyl sulfide) were dissolved separately in olive oil. Rapid injection of the TMS solution into the $\mathrm{PbO}$ solution resulted in development of the NPs. By varying growth time and concentration of TMS NPs of a specific size could be produced. The PbS NPs were precipitated by the addition of anhydrous 20 acetone and subsequent centrifugation. The material obtained was then re-dissolved in dry toluene and re-precipitated as before. It was found that the long-chain olive oil capping groups of the asprepared samples were highly insulating and so unsuitable for a device requiring charge injection into or extraction from the NP.

${ }_{25}$ Exchanging these ligands for butylamine or 3-mercaptopropionic acid resulted in samples free from charging effects during the photoemission experiments indicating that charge transport to and from the NP was possible.

For the transient absorption experiments the dynamics of the 30 lowest energy (1S) absorption peak were measured using a homebuilt transient absorption spectrometer described previously. ${ }^{5}$ In short, a white light continuum probe beam was a generated in a 2 mm-thick sapphire window by $0.8-2 \mu \mathrm{J}$ of the output of a Ti:Sapphire ultrafast regenerative amplifier operating at a centre 35 wavelength of $800 \mathrm{~nm}$ with $\sim 100 \mathrm{fs}$ pulses and a repetition rate of $1 \mathrm{kHz}$. The NP samples, in hexane or chloroform, were contained in $10 \mathrm{~mm}$ path length quartz cuvettes, and were stirred using a magnetic stirrer (Thermo Scientific Variomag Mini) at $1000 \mathrm{rpm}$ unless otherwise stated. For probe wavelengths less than $1.2 \mathrm{eV}$ 40 the probe beam was split into reference and sample beams which were balanced, in the absence of any sample photo-excitation, using a variable neutral density filter wheel before being passed through a monochromator and detected with a pair of silicon photodiodes. For probe wavelengths over $1.2 \mathrm{eV}$, beyond the ${ }_{45}$ spectral range of the silicon photodiodes, the entire probe beam passed through the sample and the monochromator and was detected by an InGaAs photodiode (FGA21, Thorlabs Inc.). The majority of the output from the regenerative amplifier was used to pump an optical parametric amplifier, and associated non-linear ${ }_{50}$ frequency conversion stages were used to generate a pump beam. The pump beam was modulated with a mechanical chopper synchronized to the 2 nd sub-harmonic of the laser repetition rate before exciting the sample. The photodiode signals were sampled using a digital lock-in amplifier and the time delay between pump 55 and probe beams was controlled by a motorized optical delay line. Four olive-oil-capped $\mathrm{PbS} \mathrm{NP}$ samples with $1 \mathrm{~S}$ absorption peaks at $1.4,1.3,1.1$ and $1.0 \mathrm{eV}$ and estimated diameters ranging from 3.4 to $4.7 \mathrm{~nm}^{26}$ were investigated. The NP samples had absorbances at the $1 \mathrm{~S}$ peak (A, calculated using the natural 60 logarithm) of $0.48,0.57,0.49$, and 0.35 respectively.

The XPS experiments were carried out both on the SuperESCA beamline at the ELETTRA synchrotron, Italy, and on the TEMPO beamline at the SOLEIL synchrotron, France. The electron energy analysers used were a hemispherical electron ${ }_{65}$ energy analyzer with $150 \mathrm{~mm}$ mean radius and a Scienta SES2002 respectively, both equipped with delay-line detectors. Photoemission spectra were recorded at room temperature and a typical total resolution of $180 \mathrm{meV}$ was estimated from a Fermi edge spectrum recorded from a clean metal surface. Four 70 butylamine-capped $\mathrm{PbS}$ nanoparticle samples with $1 \mathrm{~S}$ absorption features (effective band gaps) of 1.7, 1.4, 1.2, and $1.0 \mathrm{eV}$ and estimated diameters of $2.7,3.3,3.8$, and $4.6 \mathrm{~nm}$ respectively ${ }^{26}$ were deposited on tin-doped indium oxide (ITO) coated glass. A 3-mercaptopropionic-acid-capped $\mathrm{PbS}$ nanoparticle sample, with 75 a $1 \mathrm{~S}$ absorption features at $1.0 \mathrm{eV}$ and an estimated diameter of $4.6 \mathrm{~nm}^{26}$ was deposited onto an m-plane $\mathrm{ZnO}$ single crystal. The spectra were referenced to a metallic Fermi edge and normalised to the incident photon energy $\left(\mathrm{I}_{0}\right)$ where appropriate. A Shirleytype background was subtracted from the photoemission spectra 80 and a sum of Gaussian and Lorentzian functions with $50 \%$ Lorentzian character was used to fit the line shapes, as recommended in the literature. ${ }^{27}$ The spin-orbit splitting of the doublets was set as $4.9 \mathrm{eV}$ for the $\mathrm{Pb} 4 \mathrm{f}$ peaks and $1.2 \mathrm{eV}$ for the $\mathrm{S} 2 \mathrm{p}$ peaks.

\section{Results and Discussion}

\subsection{Multiple Exciton Generation}

Figure 1 compares the fractional change in transmission, $\Delta \mathrm{T} / \mathrm{T}$, transients induced in the $1.3 \mathrm{eV}$ band gap sample by pump 90 photons of 2.5 and $5.2 \mathrm{eV}$, i.e. 1.9 and $4.0 \times \mathrm{E}_{\mathrm{g}}$ respectively. It can be seen that when pumping with $1.9 \times \mathrm{E}_{\mathrm{g}}$ photons, when there is insufficient photon energy for MEG to occur, there are no features other than a long-lived decay. The single-exciton lifetime of olive-oil-capped PbS NPs is approximately $1 \mu \mathrm{s},{ }^{23}$ much 95 longer than the $500 \mathrm{ps}$ time window monitored in this work, and thus the signal associated with a single-exciton decay appears as a

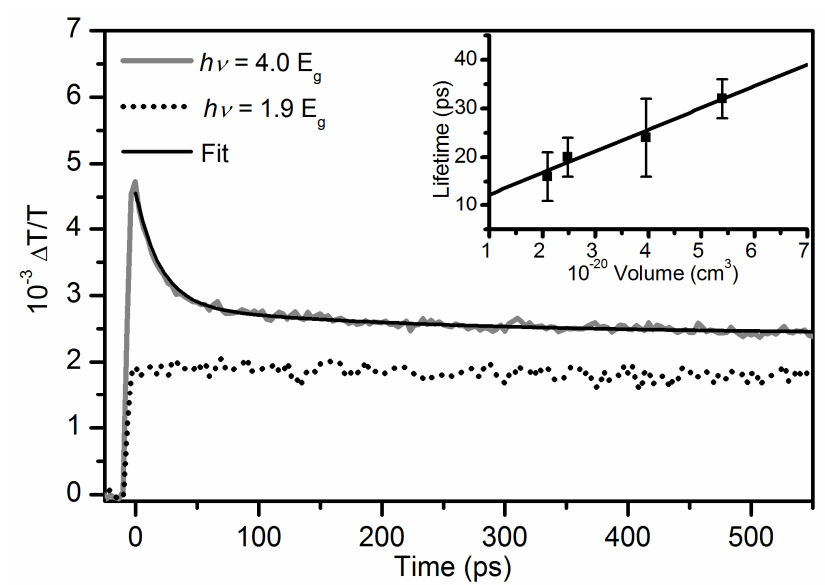

Fig. 1 Main panel: Transmittance transients induced in the $\mathrm{Eg}=1.3 \mathrm{eV} \mathrm{NP}$ sample by pumping at a photon energies corresponding to 1.9 and $4.0 \times \mathrm{E}_{\mathrm{g}}$. 100 Inset: Biexciton lifetime as a function of NP volume. 
flat plateau. In contrast, when a NP absorbs more than one photon, a biexciton decay transient in addition to the singleexciton plateau is observed ${ }^{7}$ due to the fast Auger recombination process. $^{28,} 29$ The absence of such a feature when using pump 5 energies of $1.9 \times \mathrm{E}_{\mathrm{g}}$ shows that for the fluences used the probability of a NP absorbing more than one photon per pulse is small. This is consistent with a calculated estimate of the average number of photons absorbed by a NP per pump pulse, $\langle\mathrm{N}\rangle$, for this transient. $<\mathrm{N}>$ can be estimated from the experimentally 10 obtained values of $\Delta \mathrm{T}(0) / \mathrm{T}$, which are approximately equal to the changes in absorbance, $\Delta \mathrm{A}$, for small values. The fractional change in absorbance, $\Delta \mathrm{A} / \mathrm{A}$, corresponds to the occupation number of the conduction band minimum (CBM) i.e. the ratio of $<\mathrm{N}>$ and the degeneracy of the conduction band minimum ${ }^{28}(8$ 15 for $\left.\mathrm{PbS}^{13}\right)$. Hence, for $\mathrm{PbS}<\mathrm{N}>$ is approximately $\left(8 \mathrm{~A}^{-1}\right) \Delta \mathrm{T}(0) / \mathrm{T}$, and thus equals 0.03 for the data shown. In contrast, the transient induced by $4.0 \times \mathrm{E}_{\mathrm{g}}$ energy photons includes, in addition to the plateau associated with the single exciton, a short-lived decay feature which is characteristic of a biexciton in such strongly 20 confined NPs. We attribute this feature to MEG for the following two reasons. Firstly $<\mathrm{N}>$ for this transient is less than 0.06 so this decay feature cannot be due to multiple absorptions per pulse. Secondly, vigorous stirring has previously been shown to effectively suppress photocharging ${ }^{15,} 16$ which can otherwise ${ }_{25}$ produce a similar decay feature. To confirm that the stirring rate used here is sufficient to suppress photocharging additional transients were obtained at a range of stirring rates (see ESI $\dagger$ ). Photocharging is not significant for all NP types, for example little difference is observed between stirred and unstirred samples 30 of InP NPs, ${ }^{5}$ but it is evident that, as with PbSe NPs, ${ }^{15-17}$ photocharging has a significant effect on the exciton dynamics of $\mathrm{PbS}$ NPs in unstirred solutions.
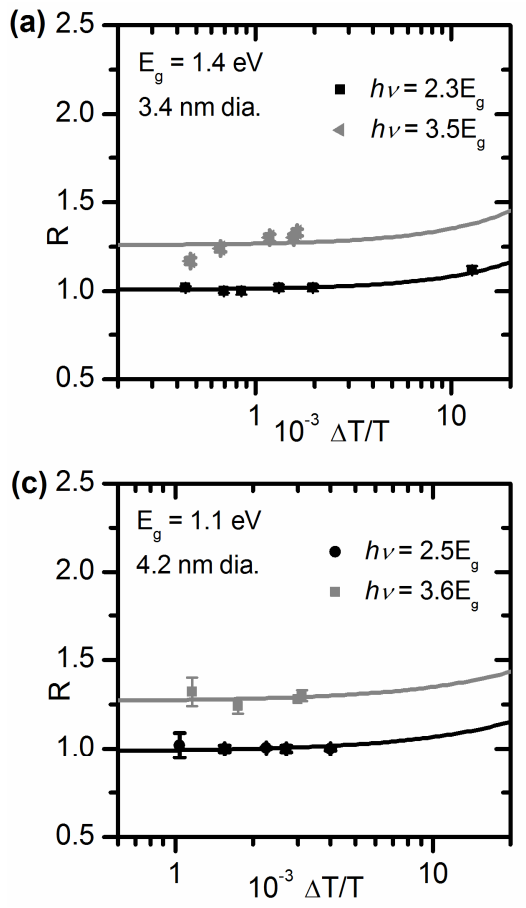

The large difference between single and biexciton lifetimes affords a means by which MEG QYs can be readily determined. ${ }_{35}$ The method of analysis used in this work, derived from earlier methods ${ }^{9,30}$ has been explained in detail by Stubbs et al. ${ }^{5}$ but is briefly described here. Small values of $\Delta \mathrm{T} / \mathrm{T}$ measured at the $1 \mathrm{~S}$ absorption peak are proportional to the exciton number density in the sample. ${ }^{31}$ The initial value of $\Delta \mathrm{T} / \mathrm{T}$ thus represents the exciton 40 number density created by the pump pulse, while its value at a time much longer than the biexciton lifetime, $\tau_{\mathrm{BX}}$, but much shorter than the single-exciton lifetime corresponds to the number of initially photoexcited NPs. The ratio of these two values, R, thus represents the average number of excitons created per 45 initially photoexcited NP, and can be found by fitting an exponential decay with a time constant of $\tau_{\mathrm{BX}}$ to the recorded transient. Most transients were well described by a single exponential but a few, particularly those obtained at high fluence and high photon energy, showed evidence of an additional slow ${ }_{50}$ decay on a timescale of approximately $10 \tau_{\mathrm{BX}}$. In these cases, the effect of this additional decay could be accounted for either by fitting a single exponential just to the first 100 ps of the transient or by fitting a biexponential decay to the whole transient and using only the component associated with $\tau_{\mathrm{BX}}$ in the calculation 55 of R. The value of $\tau_{\mathrm{BX}}$ found for each NP is plotted against NP volume (assuming a spherical shape) in the inset of Figure 1. The biexciton lifetime is seen to increase linearly with volume, in agreement with results reported previously and is attributed to the enhancement of Auger recombination by quantum confinement. ${ }_{60}$ In the limit of vanishing fluence, the probability of a NP absorbing more than one photon is negligible and thus at this limit, $R$ equals the average number of excitons created per absorbed photon i.e. the MEG QY. Figure 2 shows $R$ as a function of initial $\Delta \mathrm{T} / \mathrm{T}$ for the four samples studied and includes

(b) 2

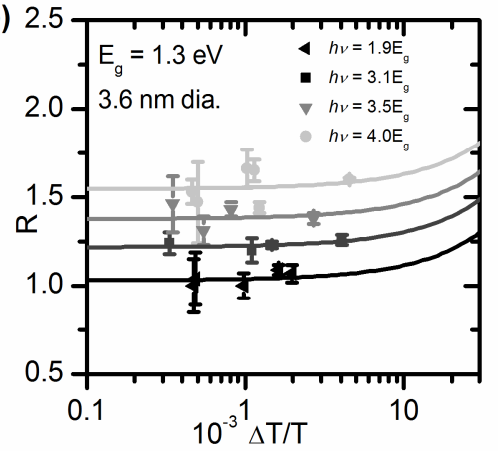

(d)

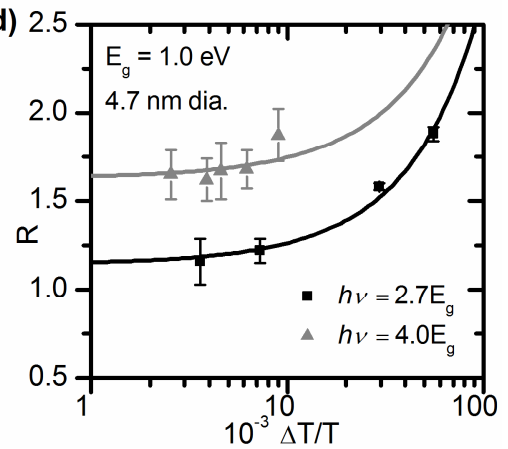

Fig.2. Average number of excitons generated per photoexcited NP, R, as function of initial fractional change in transmission, $\Delta \mathrm{T} / \mathrm{T}$, for samples with band gaps, Eg, of: (a) $1.4 \mathrm{eV}$ excited with pump photon energies 2.3 (black) and 3.5 (grey) times Eg, (b) $1.3 \mathrm{eV}$ excited with pump photon energies 1.9 (black), 3.1 (dark grey), 3.5 (grey) and 4.0 (light grey) times Eg, (c) $1.1 \mathrm{eV}$ excited with pump photon energies 2.5 (black) and 3.6 (grey) times Eg, (d) $1.0 \mathrm{eV}$ excited with pump photon energies 2.7 (black) and 4.0 (grey) times Eg. The lines shown are fitted to equation (1) with free parameter QY. 


\section{Cite this: DOI: $10.1039 / \mathrm{c0xx} 00000 x$}

fits to the following expression: ${ }^{5}$

$$
R=k \frac{\Delta T(0)}{T}\left[1-\exp \left(-\frac{k}{Q Y} \frac{\Delta T(0)}{T}\right)\right]^{-1}
$$

where $k=8 A^{-1}$ and is a constant. In all cases where the photon energy is less than $\sim 2.5 \times \mathrm{E}_{\mathrm{g}}, R$ tends to unity as $\Delta \mathrm{T} / \mathrm{T}$ decreases. 5 However, when the photon energy is more than $\sim 2.5 \times \mathrm{E}_{\mathrm{g}}, R$ is greater than unity at levels of fluence where the probability of biexciton production by multiple photon absorption is insignificant, indicating that MEG occurs at these pump energies.

Figure 3 shows the number of additional excitons created by $10 \mathrm{MEG}$, i.e. QY-1, against pump photon energy normalised to the band gap. Assuming the excess photon energy, $h v-\mathrm{E}_{\mathrm{g}}$, is partitioned between the electron and hole according to their relative effective masses, the threshold photon energy required for MEG to occur, $h v_{\text {th }}$, is given by: ${ }^{32}$

$$
h v_{t h}=E_{g}\left(2+\frac{m_{e}^{*}}{m_{h}^{*}}\right)
$$

for $m_{h}{ }^{*}>m_{e}{ }^{*}$, where $m_{e}{ }^{*}$ and $m_{h}{ }^{*}$ are the electron and hole effective masses respectively. For bulk PbS the ratio of $m_{h}{ }^{*} / m_{e}{ }^{*}$ has been reported to be approximately $0.6,{ }^{33}$ using this value the threshold photon energy for PbS NPs is expected to be $\sim 2.6 \times \mathrm{E}_{\mathrm{g}}$.

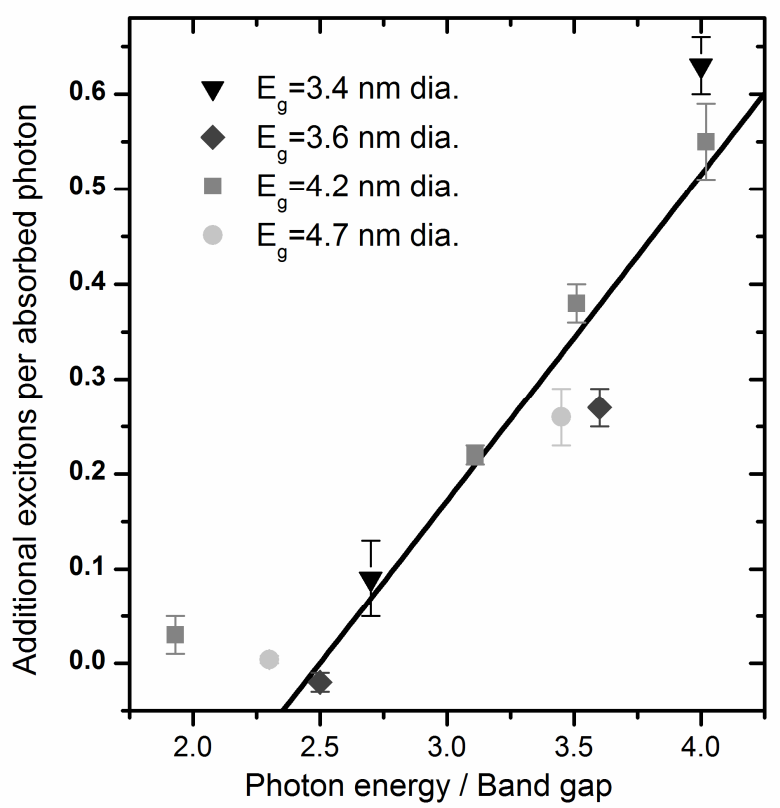

Fig.3. The additional number of excitons produced per absorbed photon by MEG as a function of hv/ $\mathrm{E}_{\mathrm{g}}$ for samples with band gaps of 1.0, 1.1, 1.3 , and $1.4 \mathrm{eV}$.

A linear fit to those points above $2.6 \times \mathrm{E}_{\mathrm{g}}$ yielded a threshold of ${ }_{25} 2.5 \pm 0.2 \times \mathrm{E}_{\mathrm{g}}$, which is consistent with the energy partition model, and a slope efficiency of $\eta=0.34 \pm 0.08$. The observation of a threshold in agreement with equation (2) strongly supports the attribution of these results to MEG rather than photocharging; only the MEG mechanism would have a threshold that is 30 dependent on $\mathrm{E}_{\mathrm{g}}$ and not just hv. Schaller et al. ${ }^{12}$ and Ellingson et $a l^{7}$ have reported results for PbS NPs consistent with a threshold of $\sim 2.6 \times \mathrm{E}_{\mathrm{g}}$ but with much greater slope efficiencies (of $\eta \sim 1.0$ ). However, both of these studies were conducted before the importance of sample stirring to avoid photocharging results was 35 known. The values of $h v_{\text {th }}$ and $\eta$ for stirred or flowed PbSe NP solutions reported recently ${ }^{15-17}$ are very similar to those found here for PbS NPs. The enhancement of photocurrent by MEG in $\mathrm{PbS}$ NPs reported by Sambur et al. ${ }^{11}$ was also found to have a threshold of $2.5 \pm 0.25 \times \mathrm{E}_{\mathrm{g}}$, although $\eta$ was measured to be

40 somewhat higher at about 0.7 . The most recently reported assessment of MEG in PbS NP ${ }^{14}$ found similar values of $h v_{\text {th }}$ and $\eta$ for NPs of approximately the same size as those studied here. Interestingly, they also reported that the value of $h v_{\mathrm{th}} / \mathrm{E}_{\mathrm{g}}$ reduced with decreasing NP size. However, as Fig. 3 shows, we observe 45 no indication of similar size dependence in this work, or of a significantly different QY for NPs of different diameters but excited at the same value of $h v / E_{g}$.

Beard et $a l .{ }^{34}$ have recently presented an analysis of MEG in which the following relationship between threshold and slope 50 efficiency was derived under the assumption that MEG is the dominant relaxation mechanism for hot electrons:

$$
h v_{t h}=E_{g}\left(1+\eta^{-1}\right)
$$

55 However, they also found that when other relaxation processes became competitive with MEG then $\eta$ is determined instead by the relative rates of MEG and these competing processes. The values for $h v_{t h}$ and $\eta$ found in this study do not satisfy Eqn. 3 indicating that in this case the rate of MEG is similar to that of 60 other relaxation processes. In contrast, the results reported by Sambur et al. ${ }^{11}$ do satisfy Eqn. 3 and thus suggest MEG is dominant. One possible explanation for this difference in behaviour, as noted by Sambur et al., ${ }^{11}$ is that the dielectric environment of the PbS NPs in the photocurrent measurements

${ }_{65}$ was significantly different from that typically used in spectroscopic measurements and that this reduced the rate of relaxation by processes other than MEG. In particular, whilst, as is the case in this study, spectroscopic measurements are usually performed on isolated NPs in an organic solvent and passivated 70 by long-chain hydrocarbons, the NPs used in the photocurrent measurements were passivated by a short-chain ligand and adsorbed onto a $\mathrm{TiO}_{2}$ crystal in an aqueous environment.

\subsection{Electronic Structure}

75 As noted above, the MEG yield may be influenced by the environment of the NPs, and the observation of MEG in solution does not guarantee that the additional carriers can be extracted 
from the NP. Of key importance to extraction are the positions of the valence band maximum (VBM) and the conduction band minimum (CBM) of the NP, relative to the surrounding photoanode and photocathode materials. The optical 5 measurements may be usefully combined with photoemission spectra to determine these frontier orbital energies as a function of nanoparticle size. Valence band spectra for 4 sizes of NP deposited on ITO substrates are shown in Fig 4. The main part of the valence band, lying between ca. 4 and $8 \mathrm{eV}$ binding energy
10 (BE) contains mainly contributions from the ligands. The features that may be ascribed to the $\mathrm{PbS}$ valence band lie at lower energies, in the range $1-5 \mathrm{eV} \mathrm{BE},{ }^{23}$ and it is here that we may expect to see the effects of quantum confinement, as the band gap, and hence the position of the valence band maximum 15 (VBM) changes with nanoparticle size. The feature at a binding energy of zero is attributed to metallic lead, arising from decomposition of $\mathrm{PbS}$ (discussed further in section 3.3) and provides a convenient reference point.

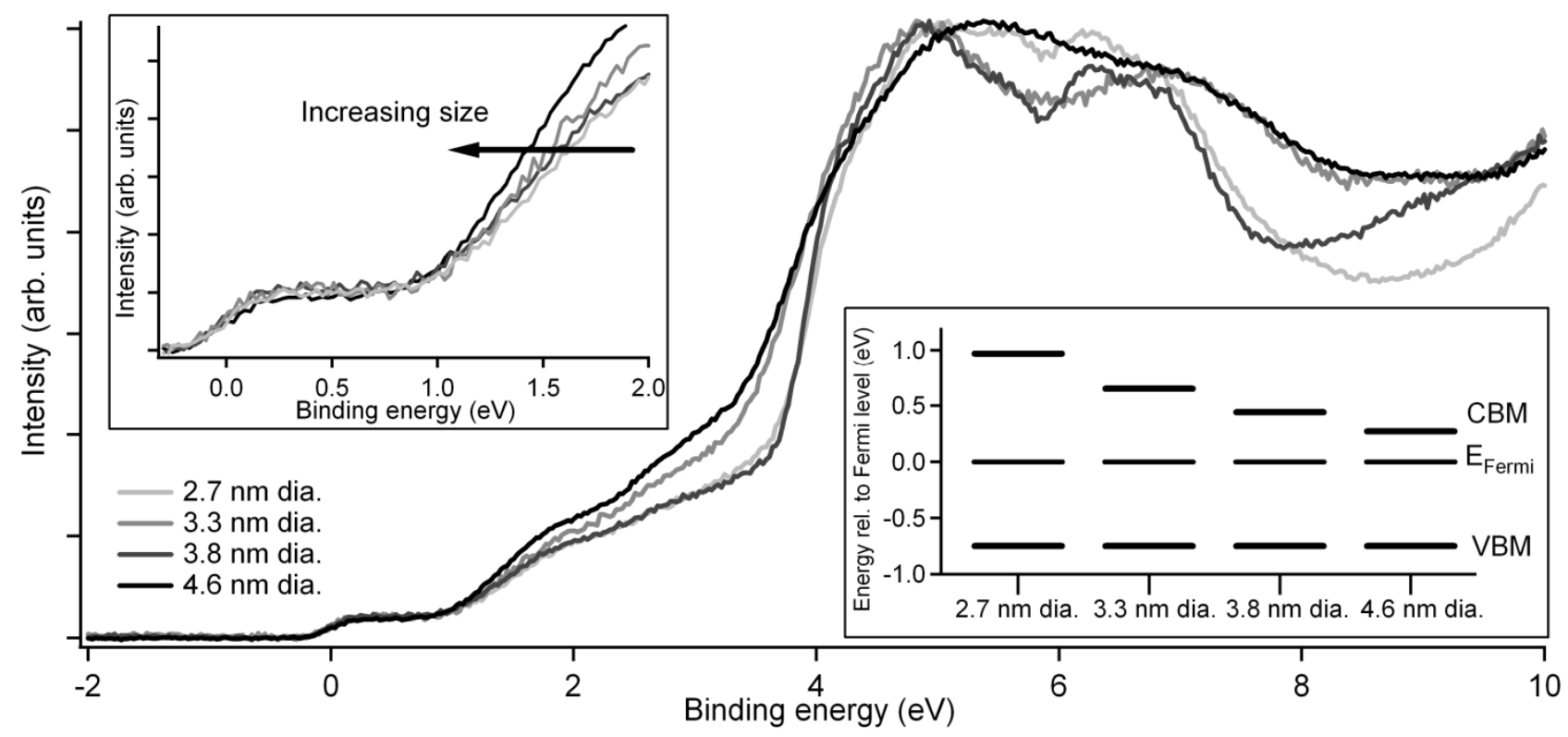

20 Fig.4. Photoemission spectra of the valence band of 4 sizes of butylamine-capped PbS nanoparticles deposited on ITO-coated glass. Insets show an enlargement of the valence band edge region and a diagram of the positions of the conduction band minimum (CBM) and valence band maximum (VBM) relative to the Fermi energy $\left(\mathrm{E}_{\mathrm{Fermi}}\right)$. The $\mathrm{CBM}$ position is inferred from optical absorption data. The $\mathrm{X}$-ray photon energy is 250 eV.

As can be seen from the figure, there is a slight shift of the valence band edge (at around $1-1.5 \mathrm{eV} \mathrm{BE}$ ) towards the Fermi 25 energy with increase in nanoparticle size (inset, Fig. 4, top). However, the change in the VBM position is within experimental error; the extrapolated VBM position is $0.75 \pm 0.1 \mathrm{eV}$ for all samples. Any shift in VBM position is at most $0.1 \mathrm{eV}$. This is very similar in magnitude to that observed by Carlson et al. ${ }^{20}$ for ${ }_{30} \mathrm{CdSe}$ nanoparticles adsorbed on $\mathrm{ZnO}$, and much smaller (by a factor of at least 10) than that expected on the basis of the simplest model of quantum confinement. This model predicts that the shifts of the VBM and the conduction band minimum (CBM) are inversely proportional to $m_{h}{ }^{*}$ and $m_{e}{ }^{*}$ respectively, and are 35 given by

$$
\Delta E_{V B M}=\frac{-h^{2}}{8 m_{h}^{*} r^{2}}, \quad \Delta E_{C B M}=\frac{h^{2}}{8 m_{e}^{*} r^{2}}
$$

where $h$ is Planck's constant and $r$ is the radius of the quantum dot. As the ratio of the hole and electron effective masses in PbS is around 0.6. ${ }^{33}$ Eqn. 4 predicts the downward shift of the CBM 40 with increasing nanoparticle size to be smaller than the upward shift of the VBM. The bottom inset to Fig. 4 shows the energy level diagrams arrived at by using the VBM measured from photoemission, together with the $1 \mathrm{~S}$ exciton energy from optical absorption measurements (taking this to give an approximate 45 measure of the position of the CBM). It is clear that the system deviates strongly from Eqn. 4, with a significant movement of the CBM, while the VBM appears to be almost pinned, analogous to the effect measured by Carlson et al. ${ }^{20}$ in CdSe. Such pinning to the VBM or Fermi level of an adjacent material requires direct ${ }_{50}$ electronic interaction, which in the case of $\mathrm{CdSe}$, is suggested to be with the $\mathrm{ZnO}$ substrate, assuming the NPs are incompletely encapsulated by the ligands. ${ }^{20}$ In our case we find that this effect is independent of the substrate used (we have repeated the experiment using a gold substrate, see ESI $\dagger$ ), and thus we believe 55 that the pinning may be due to electronic interaction with the ligand (or surface degradation products - see section 3.3) rather than the substrate. One consequence is that as the diameter of the nanoparticles increases the Fermi level moves relatively closer to the conduction band than the valence band, and thus the ${ }_{60}$ nanoparticles become effectively more n-type as the diameter increases, tending towards behaviour of bulk PbS. ${ }^{35}$ The VBM pinning has no influence on the MEG threshold (which is dependent on the band gap), or its measurement in solution for isolated NPs here. However, it may effect the overall yield and ${ }_{65}$ hence $\eta$ if the interface interaction leads to the creation of new surface states that affect the hot carrier relaxation rate, ${ }^{25}$ as discussed in section 3.1. The surface properties of the nanoparticles are therefore examined below in section 3.3. More 
importantly, the position of the CBM is crucial to the subsequent electron extraction from the nanoparticle, as the photoexcited carriers must be injected from this level into (typically) the conduction band of an n-type oxide such as $\mathrm{ZnO}$ or $\mathrm{TiO}_{2}$. Thus 5 optimisation of the CBM position is just as important as optimisation of the QY for MEG. The VBM pinning observed in this work (Fig. 4) suggests carrier injection will be significantly less efficient than expected on the basis of an effective mass model for nanoparticles with diameters larger than around $3 \mathrm{~nm} .{ }^{36}$

\subsection{Surface chemistry}

A further effect that may impair the efficiency of carrier extraction (and also the MEG yield) is the surface chemistry of the nanoparticles. Here we examine the surface chemistry of the 15 nanoparticles described in section 3.2, and how this evolves with time. Four sizes of butylamine-capped nanoparticle were deposited onto ITO-coated glass, and transferred to a UHV environment within a few hours. Using an X-ray photon energy of $250 \mathrm{eV}$, corresponding to a photoelectron inelastic mean free 20 path of $0.55 \mathrm{~nm}^{37}$ and a corresponding sampling depth of 1.65 $\mathrm{nm}$, the $\mathrm{Pb} 4 \mathrm{f}$ and $\mathrm{S} 2 \mathrm{p}$ core level spectra shown in Fig. 5 (a) - (h) were recorded. It can be seen that all the spectra are complex, arising in each case from several sets of doublets associated with different chemical species. The $\mathrm{S} 2 \mathrm{p}$ core level shows particularly

25 large chemical shifts, such that any structure observed between ca. $165 \mathrm{eV} \mathrm{BE}$ and $171 \mathrm{eV}$ BE may be attributed to oxidised species (such as sulfate and sulfite). It is immediately clear, therefore, that oxidised species are found in all the samples. The species present are assigned as neutral lead, neutral sulfur, lead 30 and sulfur as found in $\mathrm{PbS}$, and lead and sulfur as found in oxidised species $\mathrm{PbSO}_{\mathrm{x}}$. The peak positions and assignments are shown in table 1 and are in agreement with previous values reported for $\mathrm{PbS}$ nanoparticles. ${ }^{22,38}$ The sampling depth used in this experiment is fairly small compared with the diameter of the 35 nanoparticles, so this experiment probes the surface layers of the nanoparticles in the main. It is clear that a significant proportion of oxidised species is found for every particle size. As the size of the nanoparticle increases, the proportion of oxide present appears to decrease somewhat (most clearly seen in the $S 2 p$ 40 spectra of Fig. 5). By normalising the spectra using the photoionisation cross sections ${ }^{39}$ it is possible to calculate the relative amount of species present in the samples. Using the $S 2 p$ peaks we find ratios of $\mathrm{PbSO}_{\mathrm{x}}: \mathrm{PbS}$ of $1.0 \pm 0.1: 1,1.0 \pm 0.1: 1$, $0.5 \pm 0.1: 1$ and $0.5 \pm 0.1: 1$ for the four samples with estimated

45 diameters of 2.7, 3.3, 3.8 and $4.6 \mathrm{~nm}$ respectively. The proportion of oxides present appears largest for the smaller nanoparticles.

This is consistent with the larger surface-to-volume ratio of these nanoparticles, assuming that we probe mainly the surface layers in this experiment. We investigate this further below by 50 varying the sampling depth in the experiment. We believe that the neutral $\mathrm{Pb}$ and $\mathrm{S}$ could arise from rapid initial photodegradation under the intense X-ray beam at SuperESCA (as we have not observed these species in experiments at lower photon density SR sources, discussed further below); however, these signals did not 55 increase further in intensity during our experiments. The presence of metallic lead provides a useful internal Fermi level reference, as seen in section 3.2. In all cases we find a $\mathrm{Pb}: \mathrm{S}$ ratio of around $3: 1$, much larger than the expected $1: 1$. This is consistent with
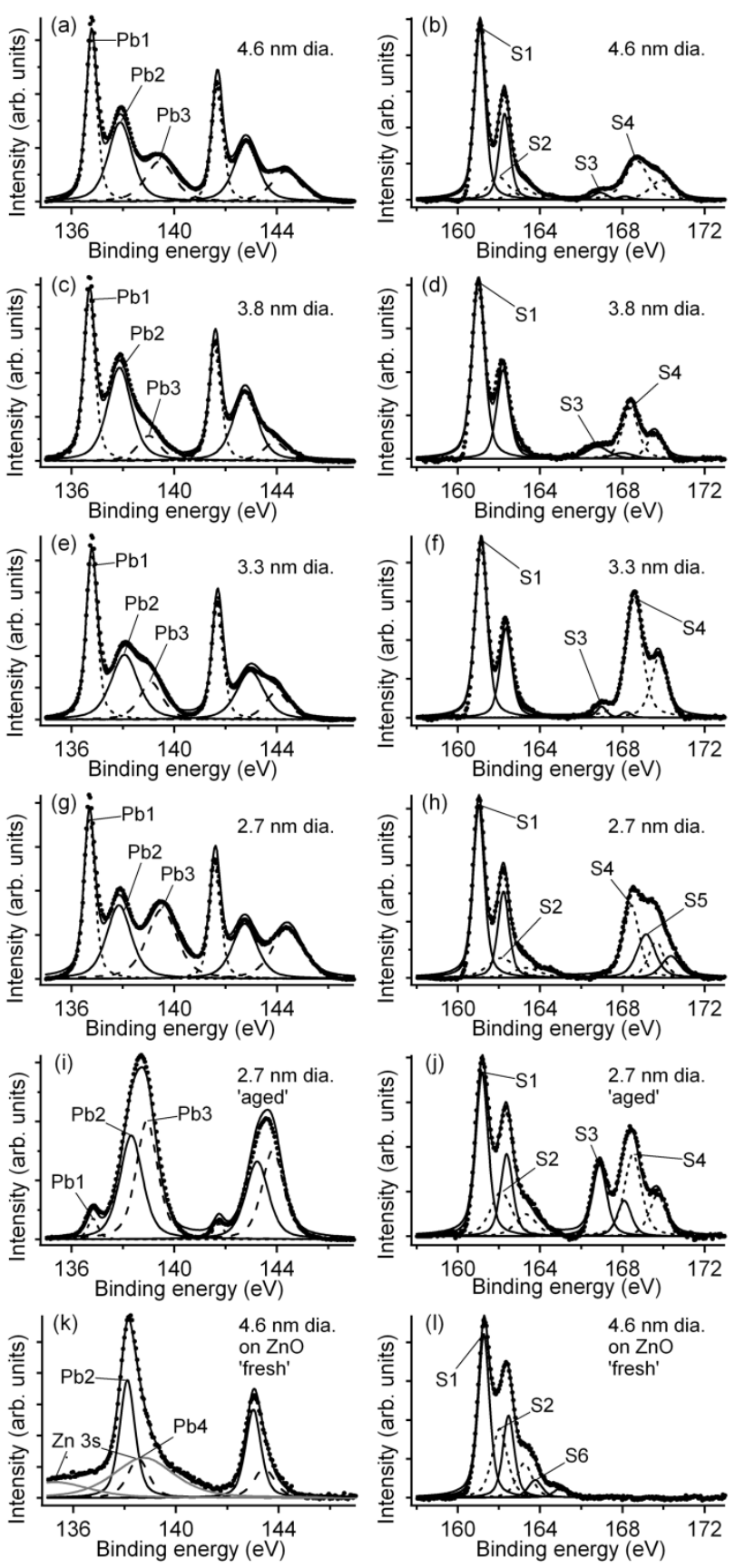

${ }_{60}$ Fig. 5. X-ray photoemission spectra $\mathrm{Pb} 4 \mathrm{f}$ (left) and $\mathrm{S} 2 \mathrm{p}$ (right) core levels for: (a) and (b) $4.6 \mathrm{~nm}$, (c) and (d) $3.8 \mathrm{~nm}$, (e) and (f) $3.3 \mathrm{~nm}$, and (g) and (h) $2.7 \mathrm{~nm}$ diameter butylamine-capped nanoparticle samples deposited on ITO-coated glass, sampled with photoelectron kinetic energy of approximately $100 \mathrm{eV}$ (photon energy $250 \mathrm{eV}$ ); (i) and (j) a $2.7 \mathrm{~nm}$

65 diameter butylamine-capped nanoparticle sample deposited on ITO coated glass which was exposed to air for ca. 1 week between deposition and study, sampled with photoelectron kinetic energy of approximately $100 \mathrm{eV}$ (photon energy $250 \mathrm{eV}$ ); (k) and (1) a $4.6 \mathrm{~nm}$ diameter 3mercaptopropionic-acid-capped nanoparticle sample deposited on $\mathrm{ZnO}$,

70 sampled with kinetic energy of approximately $80 \mathrm{eV}$ (photon energy 230 $\mathrm{eV})$. Species present are neutral lead ( $\mathrm{Pb} 1$, long dashes), lead as found in $\mathrm{PbS}(\mathrm{Pb} 2$, line), lead as found in $\mathrm{PbSOx}$ ( $\mathrm{Pb} 3$, dots), lead attached to the capping ligand $(\mathrm{Pb} 4$, dots), sulfur as found in $\mathrm{PbS}(\mathrm{S} 1$, line $)$, neutral sulfur (S2, dots), sulfur as found in oxidised PbSOx (S3, line, S4, dots, 75 and S5, line), and sulfur as found in the capping ligand (S6, dashes). ${ }^{22,38,}$ 40,4 
photodegradation of $\mathrm{PbS}$ to $\mathrm{Pb}$, with the more volatile $\mathrm{S}$ being lost to the vacuum.

Table 1. Components of $\mathrm{Pb} 4 \mathrm{f}$ and $\mathrm{S} 2 \mathrm{p}$ core level peak fitting with assignment and binding energy. ${ }^{22,38,40,41}$

\begin{tabular}{ccc}
\hline Component & Assigment & $\begin{array}{c}\text { Binding Energy (eV) } \\
\pm 0.1 \mathrm{eV}\end{array}$ \\
\hline $\mathrm{Pb} 1$ & neutral $\mathrm{Pb}$ & $136.8 \& 141.7$ \\
$\mathrm{~Pb} 2$ & lead in PbS & $138.0 \& 142.9$ \\
$\mathrm{~Pb} 3$ & lead in PbSOx & $139.1 \& 144.0$ \\
$\mathrm{~Pb} 4$ & lead in PbS linked to capping ligand & $138.5 \& 143.4$ \\
& & \\
$\mathrm{~S} 1$ & sulfur in PbS & $161.1 \& 162.3$ \\
$\mathrm{~S} 2$ & neutral sulfur & $162.0 \& 163.2$ \\
$\mathrm{~S} 3$ & sulfur in PbSOx(sulfite) & $166.9 \& 168.1$ \\
$\mathrm{~S} 4$ & sulfur in PbSOx (sulfate) & $168.6 \& 169.8$ \\
$\mathrm{~S} 5$ & sulfur in PbSOx & $169.2 \& 170.4$ \\
$\mathrm{~S} 6$ & capping ligand & $163.8 \& 165.0$
\end{tabular}

The reaction of nanoparticle surfaces with the atmosphere is an effect which has been previously observed in $\mathrm{PbS}^{22}$ To investigate this, we deposited a sample of the $2.7 \mathrm{~nm}$ butylaminecapped nanoparticles onto a substrate approximately one week 10 before the experiments took place, and then stored the sample in air. The $\mathrm{Pb} 4 \mathrm{f}$ and $\mathrm{S} 2 \mathrm{p}$ core level spectra for this sample are shown in Fig. 5(i) - (j). In comparison to the spectra of the same sample without a long exposure to air, shown in Fig. 5(g) - (h), it is clear that significant surface oxidation has occurred (an 15 increase in components $\mathrm{Pb} 3, \mathrm{~S} 3,4$, Table 1). Using the $\mathrm{S} 2 \mathrm{p}$ peaks we find the ratio of $\mathrm{PbSO}_{\mathrm{x}}: \mathrm{PbS}$ of $1.2 \pm 0.1: 1$ after ageing (Fig. $5(\mathrm{j})$ ), compared to $1.0 \pm 0.1: 1$ for the sample transferred to UHV in a few hours (Fig. 5(h)). The proportion of neutral lead is also significantly lower after ageing, perhaps suggesting that the 20 presence of surface $\mathrm{PbSO}_{4}$ passivates the $\mathrm{PbS}$ against photodegradation. Overall the results suggest that the nanoparticles become oxidised in a relatively short period on exposure to air.

In our work, we have also investigated routes to preparing $\mathrm{PbS}$ 25 nanoparticles that are chemically bound to a suitable n-type oxide, such as $\mathrm{ZnO}$, into which photoexcited electrons may be injected. Here, after synthesis in olive oil, ligand exchange was carried out with 3-mercaptopropionic acid in order to chemically link the nanoparticles to a $\mathrm{ZnO}$ single crystal substrate, using the ${ }_{30}$ approach of Carlson et al. ${ }^{20} \mathrm{The} \mathrm{Pb} 4 \mathrm{f}$ and $\mathrm{S} 2 \mathrm{p}$ core level spectra are shown in Fig. 5(k) - (1). A Zn 3s peak from the substrate is also visible in the $\mathrm{Pb} 4 \mathrm{f}$ region. In marked contrast to the butylamine-capped samples there are no signals due to any oxidised species (particularly clear in the $S 2 p$ spectrum, Fig.

35 5(1)). The speed of deposition is thought to be the main contributing factor to this, as the deposited sample was exposed to air for less than a minute before insertion into UHV. By comparing with data for the butylamine-capped samples (Fig. 5), it appears that significant oxidation occurs after aerial exposures 40 of less than an hour. In addition to the lead and sulfur species attributed to the $\mathrm{PbS}$, there are also species present attributed to $\mathrm{S}$ in the capping group, ${ }^{42} \mathrm{~Pb}$ on the surface of the nanoparticle linked to the capping group, ${ }^{22}$ and also some neutral $\mathrm{S}$ (Table 1). However, there is a striking absence of neutral $\mathrm{Pb}$. These data 45 were acquired at the TEMPO beamline at SOLEIL, using lower photon density than at SuperESCA, so less photodegradation may have occurred. However, we cannot rule out the possibility that neutral $\mathrm{Pb}$ is a product of surface ageing, along with oxidised species.
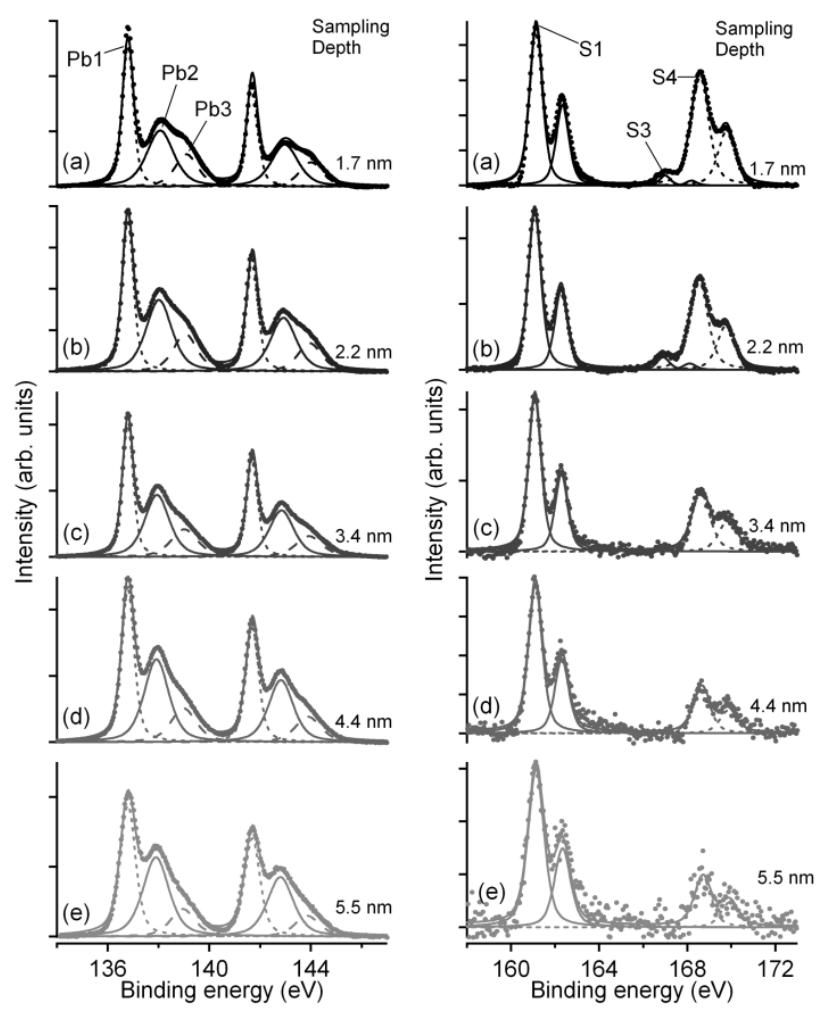

Fig. 6. X-ray photoemission spectra of $\mathrm{Pb} 4 \mathrm{f}$ (left) and $\mathrm{S} 2 \mathrm{p}$ (right) core levels for the $3.3 \mathrm{~nm}$ diameter nanoparticle sample deposited on ITOcoated glass at kinetic energies of approximately 100 (a), 200 (b), 400 (c), 600 (d) and 800 (e) eV (photon energies 250, 350, 550, 750 and $950 \mathrm{eV}$

55 respectively). Species present are neutral lead ( $\mathrm{Pb} 1$, long dashes), lead as found in $\mathrm{PbS}(\mathrm{Pb} 2$, line), lead as found in $\mathrm{PbSOx}(\mathrm{Pb} 3$, dots), sulfur as found in $\mathrm{PbS}(\mathrm{S} 1$, line), and sulfur as found in $\mathrm{PbSOx}$ (S3, line and S4, dots).

We may probe the distribution of the products of nanoparticle 60 ageing within the nanoparticle by varying the incident photon energy in photoemission experiments, and hence the photoelectron kinetic energy, which determines the photoelectron inelastic mean free path. The sampling depth from which $95 \%$ of the detected electrons originate is approximately 3 times the ${ }_{65}$ inelastic mean free path. ${ }^{43}$ Photoemission spectra of the $\mathrm{Pb} 4 \mathrm{f}$ and S $2 p$ core levels at varying incident photon energies for the 3.3 $\mathrm{nm}$ diameter nanoparticle sample (used to generate Fig. 5(e) and (f)) are shown in Fig. 6. The kinetic energy of the detected electrons ranged from 100 to $800 \mathrm{eV}$, which gives inelastic mean 70 free path values from 0.55 to $1.82 \mathrm{~nm},{ }^{37}$ and corresponding sampling depths between 1.65 and $5.46 \mathrm{~nm}$. The species present are lead and sulfur as found in $\mathrm{PbS}$, lead and sulfur as found in PbSOx, and also a large proportion of neutral lead (Table 1). On increasing the sampling depth the $\mathrm{Pb}: \mathrm{PbS}$ ratio remains 75 approximately constant at around 1:1, but the percentage of sulfur and lead present as oxidised components reduces by around 50\% over the full range of depths probed, suggesting that these oxidised components are present at the nanoparticle surfaces (Figure 6 and Table $2^{44}$ ). The ratio of $\mathrm{Pb}: \mathrm{S}$, using only the signals 80 originating from $\mathrm{PbS}$, varied from $2.4 \pm 0.1: 1$ at the smallest 
sampling depth to $1.2 \pm 0.1: 1$ at the largest. As the flux of photoelectrons is attenuated according to the Beer-Lambert law as it emerges from the sample, it is possible to obtain an estimate of the thickness of the oxidised layer by a simple model ${ }^{45}$ using 5 the integrated peak intensities and inelastic mean free paths. ${ }^{37} \mathrm{~A}$ simple 'two-layer' model, where the oxidised species (assumed to be sulfate) are assumed to form a uniform layer only at the surface gives a value of $0.5 \pm 0.2 \mathrm{~nm}$ for the depth of the oxide layer. This is significant compared with the diameter of the core 10 of the nanoparticle $(3.3 \mathrm{~nm})$.

Table 2. Ratio of the concentrations of oxidised to non-oxidised species, $[\mathrm{PbSOx}] /[\mathrm{PbS}]$, obtained from $\mathrm{S} 2 \mathrm{p}$ and $\mathrm{Pb} 4 \mathrm{f}$ core levels at a range of sampling depths for the $3.3 \mathrm{~nm}$ diameter nanoparticle sample deposited on ITO-coated glass.

\begin{tabular}{ccc}
\hline \multirow{2}{*}{$\begin{array}{c}\text { Photon energy } \\
\text { (Sampling depth) }\end{array}$} & \multicolumn{2}{c}{$[\mathrm{PbSOx}] /[\mathrm{PbS}]$} \\
\cline { 2 - 3 } & $\mathrm{S} \mathrm{2p}$ & $\mathrm{Pb} 4 \mathrm{f}$ \\
\hline $250 \mathrm{eV}(1.7 \mathrm{~nm})$ & $1.0 \pm 0.1$ & $0.5 \pm 0.1$ \\
$350 \mathrm{eV}(2.2 \mathrm{~nm})$ & $0.8 \pm 0.1$ & $0.5 \pm 0.1$ \\
$550 \mathrm{eV}(3.4 \mathrm{~nm})$ & $0.6 \pm 0.1$ & $0.5 \pm 0.1$ \\
$750 \mathrm{eV}(4.4 \mathrm{~nm})$ & $0.4 \pm 0.1$ & $0.4 \pm 0.1$ \\
$950 \mathrm{eV}(5.5 \mathrm{~nm})$ & $0.3 \pm 0.2$ & $0.3 \pm 0.1$
\end{tabular}

In summary, the $\mathrm{PbS}$ nanoparticles undergo rapid degradation on exposure to air, forming a surface layer of $\mathrm{PbSOx}$ (typically sulfate with some sulfite) that is thick compared with typical nanoparticle diameters. In the case of the $3.3 \mathrm{~nm}$ nanoparticle 20 shown in Figure 6, approximately two-thirds of the volume of the nanoparticle has been converted to oxidised species after a few hours' exposure to air. The degradation happens rapidly, on timescales of minutes, but continues on timescales of weeks. This rapid and very significant oxidative degradation is strikingly 25 similar to that observed recently in $\mathrm{PbSe}^{24}$ where a $50 \%$ conversion to oxide was observed after exposure to air for 24 hours. The formation of a substantial insulating layer at the surface of the nanoparticles might be expected to be undesirable for efficient charge extraction, but in fact has been recently 30 observed to suppress charge carrier recombination, reducing charge loss through this route. ${ }^{21}$ However, it may undesirable for the long-term stability of devices based on $\mathrm{PbS}$, unless the oxide layer is conformal around the core, passivating the surface. It also means that as a function of time, the central $\mathrm{PbS}$ core of the

35 nanoparticle will contract in diameter as its surfaces oxidise. If this happens, its band gap will increase (as has been observed in $\mathrm{PbSe}^{24}$ ), and the efficiency of MEG (for a fixed input photon energy) will decrease, as the threshold for MEG is determined by the band gap. We have observed a shift of the VBM to higher 40 binding energy, consistent with an increase in band gap, for the $2.7 \mathrm{~nm}$ 'aged' NP discussed earlier (see ESI $\dagger$ ). Following ligandexchange for photoemission, the NPs are unfortunately highly insoluble and therefore not amenable to further absorption measurements. However, we have consistently observed a blue${ }_{45} \mathrm{shift}$ in the $1 \mathrm{~S}$ absorption threshold for the initially-synthesised olive-oil-capped NPs, when stored in solution for extended periods (see ESI $\dagger$ ). At the very least, the rapid surface degradation of the nanoparticles adds some ambiguity to the determination of nanoparticle size and the correlation of this with 50 optical properties including the threshold for MEG. We suggest that this factor (in addition to experimental issues such as stirring speed) may contribute to the spread of exciton multiplicity values previously observed.

\section{$55 \quad$ 4. Conclusions}

We have presented a study of the MEG efficiency of PbS NPs in which the samples have been stirred to avoid photocharging. The values of MEG quantum yield and slope efficiency thus found are smaller than those reported by several previous spectroscopic 60 studies undertaken before the importance of photocharging was understood, and are similar to those found recently for PbSe NP samples that have also been stirred or flowed. ${ }^{15-17}$ The slope efficiency was also less than both that reported in a recent study using photocurrent measurements ${ }^{11}$ and that predicted by an ${ }_{65}$ analysis $^{34}$ under the assumption that MEG is the dominant cooling process for hot electrons. We attribute the reduced efficiency in our case to the chemical and electronic environment experienced by the NPs introducing alternate electron cooling pathways which are competitive with MEG. We have 70 investigated the electronic structure and chemistry of the interface between the PbS NPs and their surroundings when adsorbed onto suitable photoanode materials (ITO and $\mathrm{ZnO}$ ), in order to investigate whether or not the additional carriers generated by MEG may be extracted efficiently. The position of the valence 75 band maximum of the PbS NPs does not change significantly with NP size, and appears to be pinned relative to the Fermi energy (we suggest, by interaction with the ligand or surface degradation products). This effect will produce carrier injection that is significantly less efficient than expected on the basis of an 80 effective mass model for NPs with diameters larger than around 3 $\mathrm{nm}$. The NPs undergo rapid degradation on exposure to air, forming a surface layer of $\mathrm{PbSO}_{\mathrm{x}}$ that is thick compared with typical NP diameters (typically of the order of $0.5 \mathrm{~nm}$ ). This may lead to an apparent rise in MEG threshold (and drop in quantum 85 yield) as a function of time, as the core of the $\mathrm{PbS}$ nanoparticle contracts. Thus we conclude that while PbS NPs show efficient MEG, work is necessary to control their surface chemistry before its benefits are fully realised.

\section{${ }_{90}$ Acknowledgements}

This work was supported by the Engineering and Physical Sciences Research Council [grant number EP/E036287/1]; and the Northwest Science Fund (NWDA). We thank Silvano Lizzit for his assistance in performing the XPS measurements at the ${ }_{95}$ SuperESCA beamline, Sincrotrone Trieste. The research leading to these results has received funding from the European Community's Seventh Framework Programme (FP7/2007-2013) under grant agreement $\mathrm{n}^{\circ}$ 226716. We acknowledge SOLEIL for provision of synchrotron radiation facilities.

100

\section{Notes and references}

${ }^{a}$ School of Physics and Astronomy and the Photon Science Institute, University of Manchester, Oxford Road, Manchester M13 9PL, UK. E-mail:samantha.hardman@manchester.ac.uk 
${ }^{b}$ Cockcroft Institute, Daresbury Science and Innovation Campus, Warrington, Cheshire WA4 4AD, UK.

${ }^{c}$ SuperESCA beamline, Sincrotrone Trieste S.C.p.A., S.S. $14 \mathrm{Km} 163.5$, 34012 Basovizza, Trieste, Italy.

$5^{d}$ TEMPO beamline, Société civile Synchrotron SOLEIL, L'Orme des

Merisiers, Saint-Aubin - BP 48, 91192 Gif-sur-Yvette, France.

${ }^{e}$ School of Chemistry, University of Manchester, Oxford Road,

Manchester M13 9PL, UK

$\dagger$ Electronic Supplementary Information (ESI) available: comparison of 10 transients recorded with stirring rates of 0,500 and $1000 \mathrm{rpm}$, valence band spectra of 'aged' and 'fresh' $\mathrm{PbS}$ nanoparticles, absorption spectra of olive-oil-capped PbS nanoparticles. See DOI: 10.1039/b000000x/

+ Present address: University of Nova Gorica Vipavska 11c, 5270 Ajdovscina, Slovenia.

$15 \S$ Present address: Nanoscience Centre and Catalysis Division, National Center for Physics (NCP), Quaid-i-Azam University, Islamabad, Pakistan

1. W. Shockley and H. J. Queisser, Journal of Applied Physics, 1961, 32, 510-519.

2. R. D. Schaller, M. A. Petruska and V. I. Klimov, Applied Physics Letters, 2005, 87, 253102

3. Y. Kobayashi, T. Udagawa and N. Tamai, Chemistry Letters, 2009, 38, 830-831.

25 4. D. Gachet, A. Avidan, I. Pinkas and D. Oron, Nano Letters, 2010, 10, 164-170.

5. S. K. Stubbs, S. J. O. Hardman, D. M. Graham, B. F. Spencer, W. R. Flavell, P. Glarvey, O. Masala, N. L. Pickett and D. J. Binks, Physical Review B, 2010, 81, 081303(R).

30 6. R. D. Schaller, J. M. Pietryga and V. I. Klimov, Nano Letters, 2007, 7, 3469-3476.

7. R. J. Ellingson, M. C. Beard, J. C. Johnson, P. R. Yu, O. I. Micic, A. J. Nozik, A. Shabaev and A. L. Efros, Nano Letters, 2005, 5, 865871.

35 8. J. E. Murphy, M. C. Beard, A. G. Norman, S. P. Ahrenkiel, J. C. Johnson, P. R. Yu, O. I. Micic, R. J. Ellingson and A. J. Nozik, Journal of the American Chemical Society, 2006, 128, 3241-3247.

9. M. C. Beard, K. P. Knutsen, P. R. Yu, J. M. Luther, Q. Song, W. K. Metzger, R. J. Ellingson and A. J. Nozik, Nano Letters, 2007, 7, $40 \quad 2506-2512$.

10. V. Sukhovatkin, S. Hinds, L. Brzozowski and E. H. Sargent, Science, 2009, 324, 1542-1544.

11. J. B. Sambur, T. Novet and B. A. Parkinson, Science, 2010, 330, 6366.

45 12. R. D. Schaller, M. Sykora, J. M. Pietryga and V. I. Klimov, Nano Letters, 2006, 6, 424-429.

13. G. Nair, S. M. Geyer, L. Y. Chang and M. G. Bawendi, Physical Review B, 2008, 78, 125325.

14. G. Nootz, L. A. Padilha, L. Levina, V. Sukhovatkin, S. Webster, L.

50 Brzozowski, E. H. Sargent, D. J. Hagan and E. W. Van Stryland, Physical Review B, 2011, 83, 7.

15. J. A. McGuire, J. Joo, J. M. Pietryga, R. D. Schaller and V. I. Klimov, Accounts of Chemical Research, 2008, 41, 1810-1819.

16. J. A. McGuire, M. Sykora, J. Joo, J. M. Pietryga and V. I. Klimov,

$55 \quad$ Nano Letters, 2010, 10, 2049-2057.

17. A. G. Midgett, H. W. Hillhouse, B. K. Hughes, A. J. Nozik and M. C. Beard, Journal of Physical Chemistry C, 2010, 114, 17486-17500.

18. D. J. Asunskis, I. L. Bolotin, J. E. Haley, A. Urbas and L. Hanley, Journal of Physical Chemistry C, 2009, 113, 19824-19829.
60 19. A. M. Munro, B. Zacher, A. Graham and N. R. Armstrong, ACS Applied Materials \& Interfaces, 2010, 2, 863-869.

20. B. Carlson, K. Leschkies, E. S. Aydil and X. Y. Zhu, Journal of Physical Chemistry C, 2008, 112, 8419-8423.

21. N. Zhao, T. P. Osedach, L.-Y. Chang, S. M. Geyer, D. Wanger, M. T.

65 Binda, A. C. Arango, M. G. Bawendi and V. Bulovic, ACS Nano, 2010, 4, 3743-3752.

22. A. Lobo, T. Moller, M. Nagel, H. Borchert, S. G. Hickey and H. Weller, Journal of Physical Chemistry B, 2005, 109, 17422-17428.

23. J. Akhtar, M. A. Malik, P. O'Brien, K. G. U. Wijayantha, R.

70 Dharmadasa, S. J. O. Hardman, D. M. Graham, B. F. Spencer, S. K. Stubbs, W. R. Flavell, D. J. Binks, F. Sirotti, M. El Kazzi and M. Silly, Journal of Materials Chemistry, 2010, 20, 2336-2344.

24. M. Sykora, A. Y. Koposov, J. A. McGuire, R. K. Schulze, O. Tretiak, J. M. Pietryga and V. I. Klimov, ACS Nano, 2010, 4, 2021-2034.

75 25. G. Allan and C. Delerue, Physical Review B, 2009, 79, 5.

26. L. Cademartiri, E. Montanari, G. Calestani, A. Migliori, A. Guagliardi and G. A. Ozin, Journal of the American Chemical Society, 2006, 128, 10337-10346.

27. R. Hesse, P. Streubel and R. Szargan, Surface and Interface Analysis, 2007, 39, 381-391.

28. V. I. Klimov, Journal of Physical Chemistry B, 2000, 104, 61126123.

29. V. I. Klimov, A. A. Mikhailovsky, D. W. McBranch, C. A. Leatherdale and M. G. Bawendi, Science, 2000, 287, 1011-1013.

85 30. R. D. Schaller and V. I. Klimov, Physical Review Letters, 2004, 92.

31. V. I. Klimov, Semiconductor and Metal Nanocrystals, Marcel Dekker, New York, 2004.

32. M. C. Beard and R. J. Ellingson, Laser \& Photonics Reviews, 2008, 2, 377-399.

90 33. D. R. Lide, ed., Handbook of Physics and Chemistry, CRC Press, Boca Raton, FL, 2009.

34. M. C. Beard, A. G. Midgett, M. C. Hanna, J. M. Luther, B. K. Hughes and A. J. Nozik, Nano Letters, 2010, 10, 3019-3027.

35. W. W. Scanlon, Journal of Physics and Chemistry of Solids, 1959, 8, $95 \quad 423-428$

36. B. R. Hyun, Y. W. Zhong, A. C. Bartnik, L. F. Sun, H. D. Abruna, F. W. Wise, J. D. Goodreau, J. R. Matthews, T. M. Leslie and N. F. Borrelli, ACS Nano, 2008, 2, 2206-2212.

37. S. Tanuma, C. J. Powell and D. R. Penn, Surface and Interface Analysis, 1991, 17, 927-939.

38. D. J. Asunskis and L. Hanley, Surface Science, 2007, 601, 46484656.

39. J. J. Yeh and I. Lindau, Atomic Data and Nuclear Data Tables, 1985, 32, 1-155.

105 40. J. Tang, L. Brzozowski, D. A. R. Barkhouse, X. H. Wang, R. Debnath, R. Wolowiec, E. Palmiano, L. Levina, A. G. PattantyusAbraham, D. Jamakosmanovic and E. H. Sargent, ACS Nano, 2010, 4, 869-878.

41. NIST XPS database, http://srdata.nist.gov/xps/, Accessed 29th September 2010.

42. G. Gonella, O. Cavalleri, S. Terreni, D. Cvetko, L. Floreano, A. Morgante, M. Canepa and R. Rolandi, Surface Science, 2004, 566568, 638-643.

43. J. C. Vickerman, ed., Surface Analysis - The Principle Techniques, 115 John Wiley \& Sons, Chichester, 1997. 
44. As the binding energies of the $\mathrm{Pb} 4 \mathrm{f}$ and $\mathrm{S} 2 \mathrm{p}$ levels are similar, the sampling depth (which differ by only around $0.1 \mathrm{~nm}$ ) is taken as the same for both levels. This assumption breaks down somewhat for the most surface sensitive measurements (at $250 \mathrm{eV}$ and $350 \mathrm{eV}$ photon energy), where the surface composition is changing rapidly as a function of depth, and a higher proportion of oxide is probed by the more surface sensitive experiment using the $S 2 p$ signal. As the sampling depth increases, the agreement between the compositions obtained from the $\mathrm{Pb} 4 \mathrm{f}$ and $\mathrm{S} 2 \mathrm{p}$ signals improves.

10 45. B. R. Strohmeier, Surface and Interface Analysis, 1990, 15, 51-56. 